\title{
Early-Warning Method of Train Running Safety of a High-Speed Railway Bridge Based on Transverse Vibration Monitoring
}

\author{
You-Liang Ding, ${ }^{1}$ Peng Sun, ${ }^{2}$ Gao-Xin Wang, ${ }^{1}$ Yong-Sheng Song, ${ }^{1}$ Lai-Yi Wu, \\ Qing Yue, ${ }^{3}$ and Ai-Qun $\mathrm{Li}^{1}$ \\ ${ }^{1}$ Key Laboratory of Concrete and Prestressed Concrete Structures of Ministry of Education, Southeast University, \\ Nanjing 210096, China \\ ${ }^{2}$ Department of Civil and Environmental Engineering, Rice University, Houston, TX 77005, USA \\ ${ }^{3}$ China Railway Major Bridge (Nanjing) Bridge and Tunnel Inspect \& Retrofit Co., Ltd, Nanjing 210032, China
}

Correspondence should be addressed to You-Liang Ding; civilchina@hotmail.com

Received 13 August 2014; Accepted 10 October 2014

Academic Editor: Bo Chen

Copyright (c) 2015 You-Liang Ding et al. This is an open access article distributed under the Creative Commons Attribution License, which permits unrestricted use, distribution, and reproduction in any medium, provided the original work is properly cited.

\begin{abstract}
Making use of long-term transverse vibration monitoring data of DaShengGuan Bridge, the early-warning method of train running safety of the high-speed railway bridge is established by adopting principal component analysis (PCA) method. Firstly, the root mean square (RMS) of the transverse acceleration of the main girder is used as the monitoring parameter for the train running safety. The correlation model between the RMS values measured from different positions is further adopted as the evaluating model for the train running safety. Finally, the effects of the environmental changes on the evaluating model are eliminated using the PCA method and the warning index for the train running safety is further constructed. The analysis results show that the correlation between the RMS values of the accelerations from different measuring positions on the main girder can be analyzed by a quadratic polynomial fitting model. The PCA method can effectively remove the environmental effects on the quadratic polynomial fitting model. The proposed warning method provides a good capability for detecting the abnormal changes of the measured transverse accelerations and hence it is suitable for early-warning of the train running safety.
\end{abstract}

\section{Introduction}

Nanjing DaShengGuan Bridge, which serves as the shared corridor crossing Yangtze River for both Beijing-Shanghai high-speed railway and Shanghai-Wuhan-Chengdu railway, is the first 6-track high-speed railway bridge with the longest span throughout the world. With its $336 \mathrm{~m}$ main span and 6track railways it ranks itself the largest bridge with heaviest design loading among the high-speed railway bridges by far. Also the design speed of $300 \mathrm{~km} / \mathrm{h}$ of the bridge is on the advanced level in the world. Due to these remarkable characteristics including long span of the main girder, heavy design loading, and high speed of trains, the considerable transverse vibration of the bridge caused by high-speed trains may threaten the train running safety of DaShengGuan Bridge. Thus the train running safety should be paid special attention during the bridge operation [1-3]. During the long-time service of the bridge, the environmental actions (wind, temperature, rain, etc.) and the bridge usage (highspeed trains) can continually modify the behavior and local damage of their key positions in the bridge may continually accumulate and cause deterioration of the bridge. In particular the deterioration of the transverse stiffness of the bridge may increase the transverse vibration of the bridge so as to threaten the train running safety. Therefore, research efforts should be focused on the transverse vibration monitoring of the bridge so as to gather reliable information that can be used to guarantee the train running safety.

To realize the transverse vibration monitoring of the bridge, the structural health monitoring system for the DaShengGuan Bridge has been established by the application 
of modern techniques in sensing, testing, computing, and network communication. The ideal objective of the bridge health monitoring system is to gather various reliable information that can be used to detect the evolution of the bridge's condition state and perform reliability-based assessment so as to give the engineer or administrant a great variety of options with respect to maintenance intervention [4-6]. However, how to explain the health condition of the bridge structure according to the collected structural responses remains a great challenge in the civil engineering community [7]. It is well known that bridge structures are subject to varying environmental conditions such as loadings and environmental temperature. These environmental effects will cause changes in the measured structural responses that may mask the changes caused by structural damage $[8,9]$. Therefore, it is of paramount importance to characterize the normal variability of structural responses due to environmental effects and discriminate such normal variability from abnormal changes in structural responses caused by the deterioration of the bridge. Considerable research efforts have been devoted to investigating the influences of environmental conditions on the measured structural responses of bridges [10-13]. For instance, $\mathrm{Ni}$ et al. [10] observed that the normal environmental change of Ting Kau Bridge accounts for relative variation in modal frequencies from $3.22 \%$ to $15.07 \%$ for the first ten modes. Using the formulated frequency-temperature correlation models, the relative variation of the normalized frequencies after removing the temperature effect was reduced to range from $1.61 \%$ to $7.87 \%$. Ni et al. [11] presented a procedure for condition assessment of expansion joints based on long-term monitoring of displacement and temperature. The results revealed that temperature fluctuation mainly accounts for the movement of the expansion joint. However, relevant works focused on the influences of environmental conditions on the measured transverse accelerations of high-speed railway bridges are seldom reported. Hence, it is necessary to present a procedure for early warning of train running safety under changing environmental conditions using the long-term transverse vibration monitoring data of high-speed railway bridges.

Based on the aforementioned motivation, the objective of this paper is to propose an early-warning method of train running safety for high-speed railway bridges. The variability of transverse accelerations of the main girder induced by high-speed trains is quantified and the abnormal changes of the measured transverse accelerations which may threaten the train running safety are detected using the principal component analysis (PCA) method. The paper emphasizes on (i) the choice of monitoring parameter obtained from the transverse accelerations of the main girder; (ii) the establishment of the evaluating model for the train running safety; and (iii) the construction of the warning index using the PCA method. The feasibility of the proposed strategy is demonstrated using 310 days of measured transverse acceleration data on the main girder of Nanjing DaShengGuan Bridge in 2013.

\section{Early-Warning Method of Train Running Safety Using Transverse Vibration Monitoring Data}

The train running safety is to refer to the safe traveling status of trains without derailment. In particular for high-speed railway bridges, high-speed train derailment incidents may cause heavy casualties and property losses. In the design of high-speed railway bridges, the derailment coefficient and wheel unloading rate are usually used to judge the train running safety. However, Zeng et al. [14] point out that the current limit values of derailment coefficient and wheel unloading rate cannot prevent the happening of derailment. The essential reason of train derailment is that the transverse vibration of the time-variant system of train-railwaybridge becomes unstable. Hence, if the system can maintain a stable transverse vibration condition, the prevention of derailment can be achieved. In the present study, the earlywarning method of train running safety of the high-speed railway bridge using transverse vibration monitoring data is established in combination with the theory of energy random analysis of railway derailment proposed by Zeng et al. [14] and structural health monitoring system of railway bridges. The early-warning method of train running safety is to monitor the transverse vibration of the main girder of the bridge. When the high-speed trains pass through the bridge, the various wheel-rail contact states can produce various transverse vibration responses in the main girder of a bridge. Whether the measured transverse vibration of the main girder is stable can indicate the transverse vibration condition of the train-railway-bridge system, which results in the judgment of train running safety. Thus, anomaly alarms for train running safety can be triggered on time if the monitoring transverse vibration data of the main girder disobey the normal pattern due to the deterioration of the bridge.

The early-warning method of train running safety using transverse vibration monitoring data involves three steps:

(1) to extract the monitoring parameter for the train running safety, that is, to extract the monitoring parameter from the transverse acceleration responses of the main girder, which can indicate the train running safety of high-speed railway bridges: this paper will investigate applicability of two monitoring parameters including the peak value and the RMS value of the transverse acceleration;

(2) to construct the evaluation model for the train running safety; due to the strong stochastic features of the transverse acceleration responses of the train-railway-bridge system with high-speed train traveling by, a deterministic evaluation model should be established to represent the stable condition of the transverse vibration for the whole system based on the extracted monitoring parameter;

(3) to establish an early-warning index for the train running safety; the stability of the transverse vibration of the train-railway-bridge system is a basic rule for the judgment of train running safety; hence, a warning index for the train running safety is further constructed from the constructed evaluation model using a statistical pattern recognition 


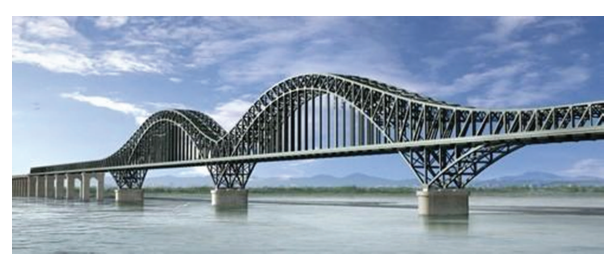

(a) View of the Nanjing DaShengGuan Bridge

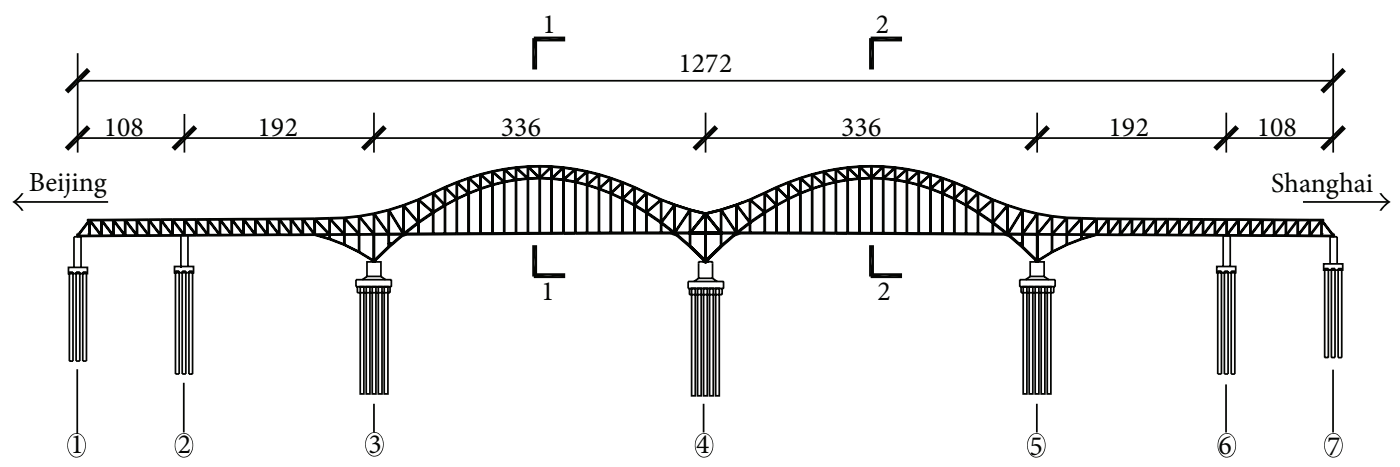

(b) Elevation drawing of the bridge (unit: $\mathrm{m}$ )

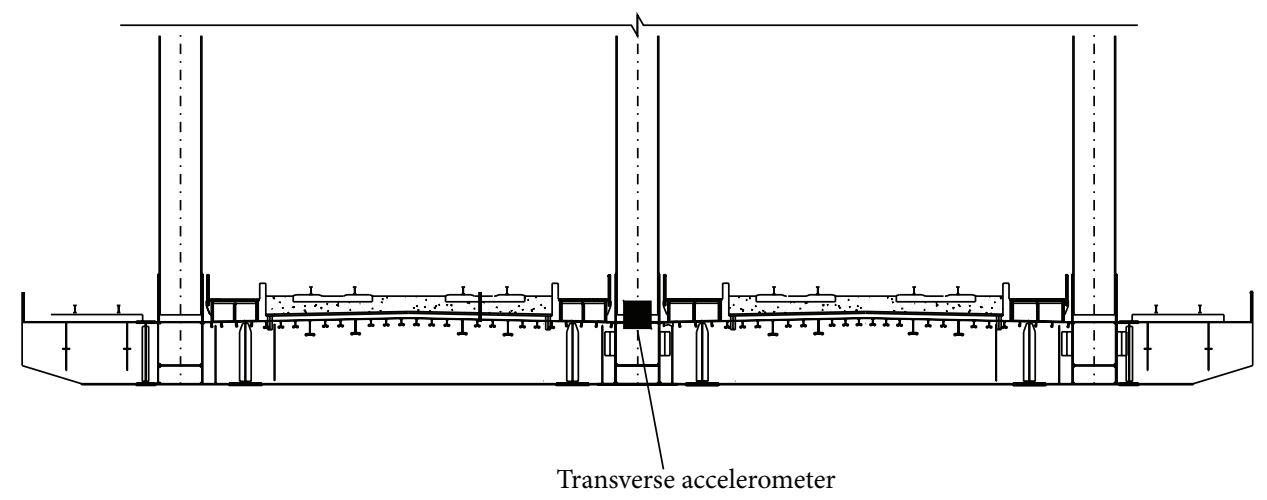

(c) Locations of the accelerometers in the deck sections

FIGURE 1: View of the Nanjing DaShengGuan Bridge and layout of the transverse vibration monitoring of the main girder.

method; the abnormal condition for the train running safety can be alarmed if the monitoring evaluation model disobeys the normal pattern.

\section{Application in Early-Warning of Train Running Safety of DaShengGuan Bridge}

3.1. Transverse Vibration Monitoring of the Girder of DaShengGuan Bridge. The subject of this study is Nanjing DaShengGuan Bridge shown in Figure 1(a), which is a steel truss arched bridge with the span arrangement $(108+192+2 \times$ $336+192+108) \mathrm{m}$. The elevation drawing of the bridge is shown in Figure 1(b). The health monitoring system for the DaShengGuan Bridge has been established to real-time monitor the responses of the bridge under the various kinds of environment actions and high-speed trains. In order to monitor the transverse acceleration responses on the two main spans induced by the traveling high-speed trains, one transverse accelerometer has been installed at the 1-1 section and 2-2 section in the middle of the two main spans, respectively, as shown in Figure 1(c). The two accelerometers are named as JSD-11-04 and JSD-15-06, respectively. The sampling frequency of two accelerometers is $200 \mathrm{~Hz}$. A total of 310 days of transverse acceleration data measured in 2013 are used in this study.

3.2. Analysis of Measured Transverse Vibration Data of the Girder of DaShengGuan Bridge. Figure 2 illustrates the typical transverse acceleration time histories of the main girder measured from two accelerometers. It can be observed that when high-speed train traveled by, there are obvious stochastic vibration features in the transverse accelerations in the middle of the two main spans of the main girder, and the transverse accelerations in the middle of the first span are much smaller than those of the second span. These indicate that although the structural layouts of two main spans of the main girder are the same, there exits significant difference between the transverse vibration characteristics of two main 


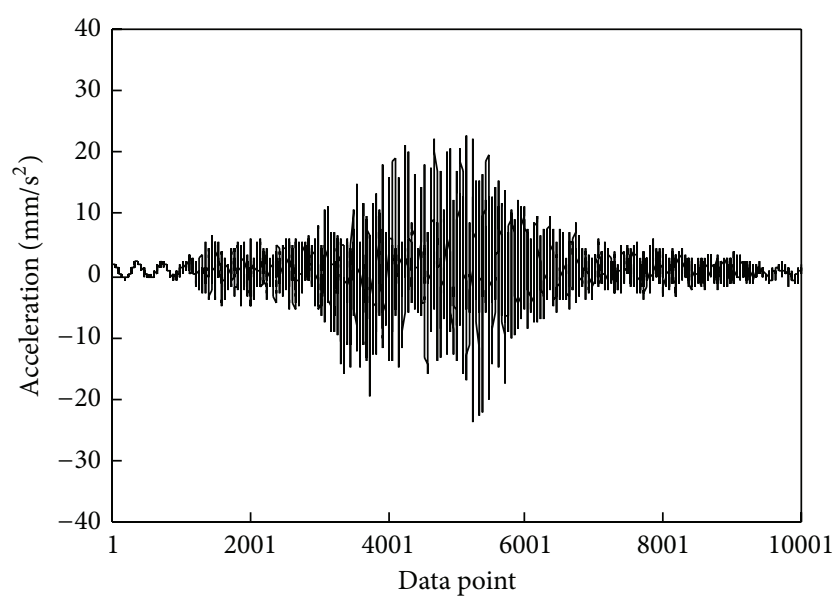

(a) Accelerometer JSD-11-04

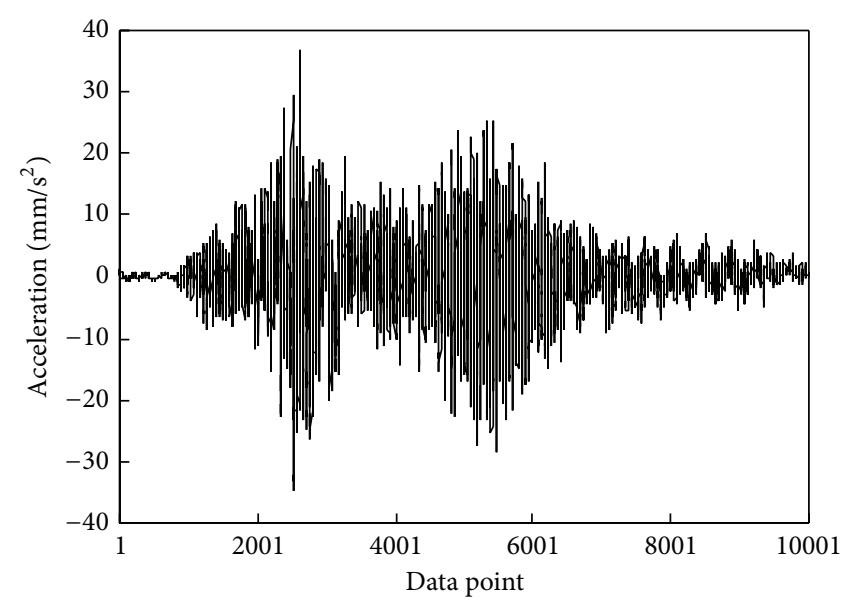

(b) Accelerometer JSD-15-06

FIGURE 2: Transverse acceleration time histories of the main girder induced by a high-speed train.

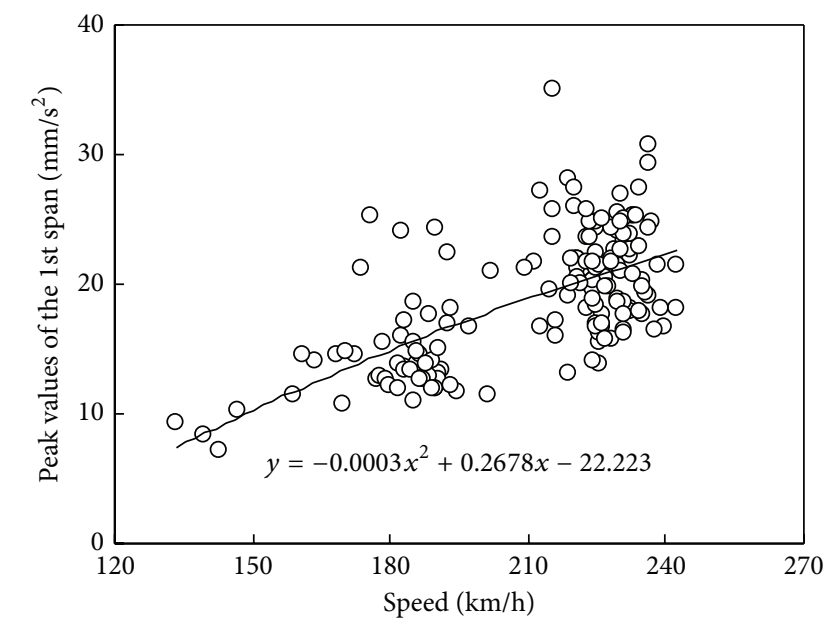

(a) Correlation between the peak value of transverse accelerations and the speed of trains

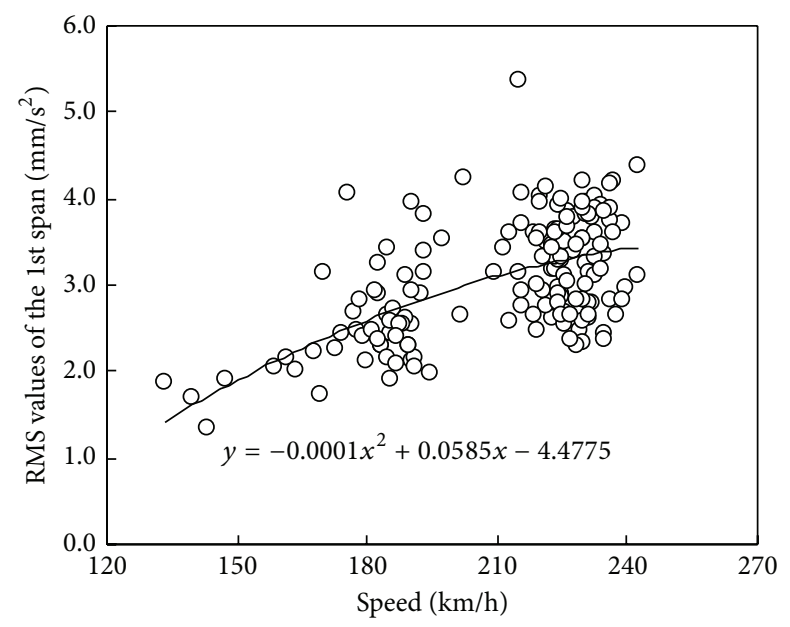

(b) Correlation between the RMS value of transverse accelerations and the speed of trains

FIGURE 3: Correlations between the amplitude parameters of the transverse accelerations in the middle of the first main span and the speed of the trains.

spans due to the rail irregularity in transverse direction. Thus, there is a need to monitor the transverse accelerations of two main spans in the long term so as to obtain the transverse vibration state of the train-railway-bridge system and realize anomaly alarms for train running safety.

In the present study, the acceleration amplitude parameters are utilized as the monitoring parameters to represent the transverse vibration characteristic of the main girder. The acceleration amplitude usually refers to the maximum or effective value of acceleration responses. For this reason, the applicability of the two acceleration amplitude parameters, the peak value and the RMS value, should be studied in this research. It should be noted that, in the calculation of RMS values of the transverse acceleration of the main girder, the filter bandwidth of the transverse accelerations is set to be $0-20 \mathrm{~Hz}$. Figure 3 shows the scatter plots between the amplitude parameters of the transverse accelerations in the middle of the first main span and the speed of the trains each time the trains passed by on February 1st, 2013. The train speed is measured using speedometer installed in the middle of the second main span of the main girder. It can be observed that, with the increase of train speed, both the peak value and the RMS value of the transverse accelerations have an upward trend. However, it is obvious that the measurement points in Figure 3 are too dispersed to be an effective description of the correlation between the amplitude parameters and train velocities, especially when the speed of trains exceeds $200 \mathrm{~km} / \mathrm{h}$. This implies that the transverse vibration responses of the main girder due to the different trains at the same speed varied much, mainly because of the various wheel-rail contact states of the trains. The analysis results indicate that it is hard to construct an efficient correlation model between the amplitude parameter and the speed of trains so as to represent the transverse vibration state 


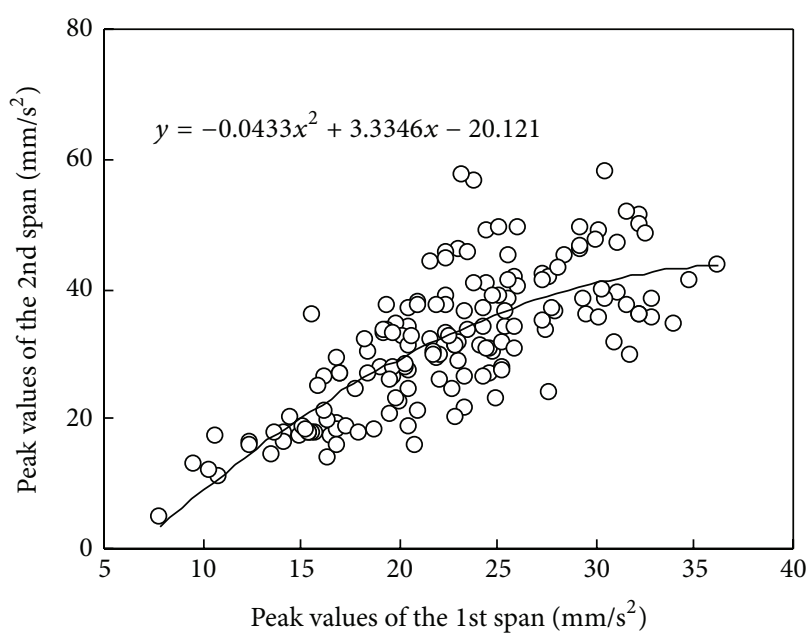

(a) Cross-correlation between peak values of transverse accelerations

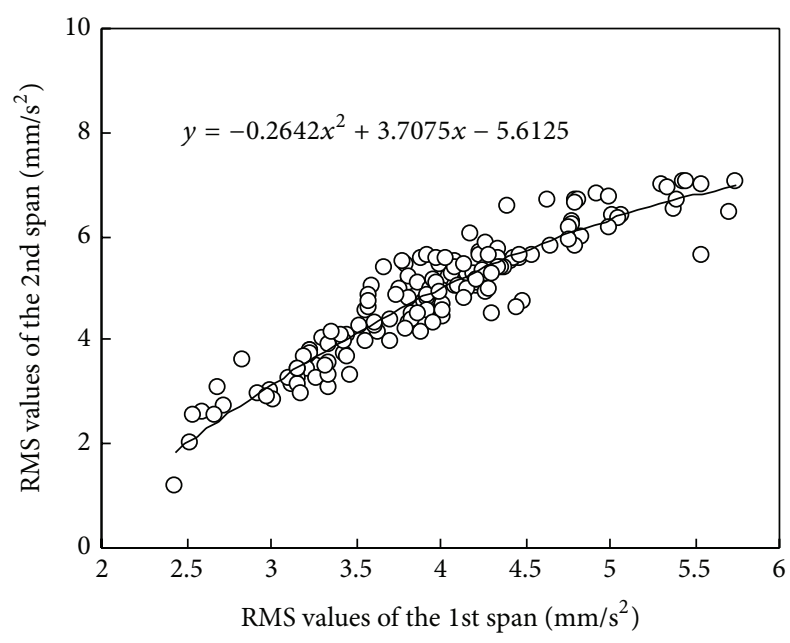

(b) Cross-correlation between RMS values of transverse accelerations

FIGURE 4: Cross-correlations between the amplitude parameters of the transverse accelerations measured in the middle of two main spans.

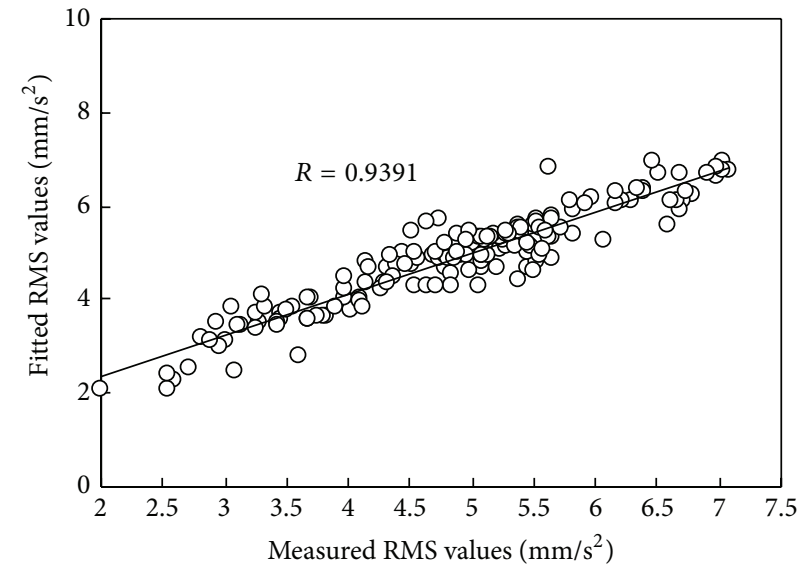

(a) Correlation between the measured RMS values of the transverse accelerations in the middle of the second span and the corresponding fitted values

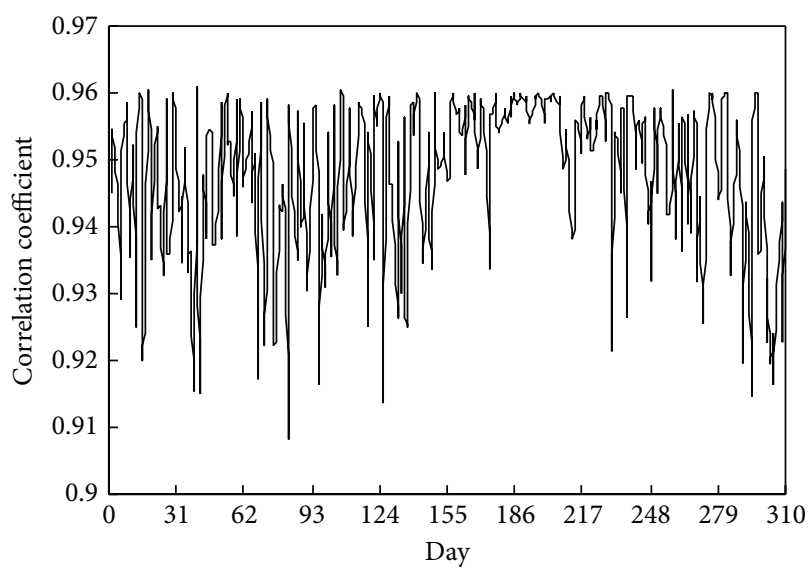

(b) Computed correlation coefficients of the 310 days in 2013

FIGURE 5: Fitting effect of cross-correlation between RMS values of transverse accelerations by using quadratic polynomial.

of the train-railway-bridge system. In addition, it can be also observed from Figure 3 that the fluctuations of the amplitude parameters of the transverse accelerations on the main girder are very high. This is due to the strong stochastic feature of the transverse vibration of the train-railway-bridge system when the high-speed trains pass through the bridge. Hence, it is also hard to construct an efficient evaluation model to represent the transverse vibration state of the train-railwaybridge system simply using transverse accelerations at a single measurement point.

In the next discussion, the cross-correlation between the amplitude parameters of the transverse accelerations in the middle of two main spans is investigated when the high-speed trains pass through the bridge. Figure 4(a) shows the scatter plots between the peak values of the transverse accelerations in the middle of two main spans on February 1st, 2013, and shows the fitted results by using quadratic polynomial as well.
Figure 4(b) shows the scatter plots between the RMS values of the transverse accelerations and shows the fitted results by using quadratic polynomial as well. By comparison, it can be concluded that the cross-correlation by using the RMS values is much better than the cross-correlation by using the peak values. By using the fitting formula constructed in Figure 4(b), scatter plot between the RMS values of the transverse accelerations in the middle of the second span and the corresponding fitted values is drawn as Figure 5(a), where the fitted values were obtained by taking the RMS values of the transverse accelerations in the middle of the first span into the fitting formula of the quadratic polynomial. The correlation coefficient of the measured RMS values and fitted values on February 1st, 2013, is 0.9391, as shown in Figure 5(a). Computed using the same method, all the correlation coefficients of the 310 days in 2013 are more than 0.90, as shown in Figure 5(b). 


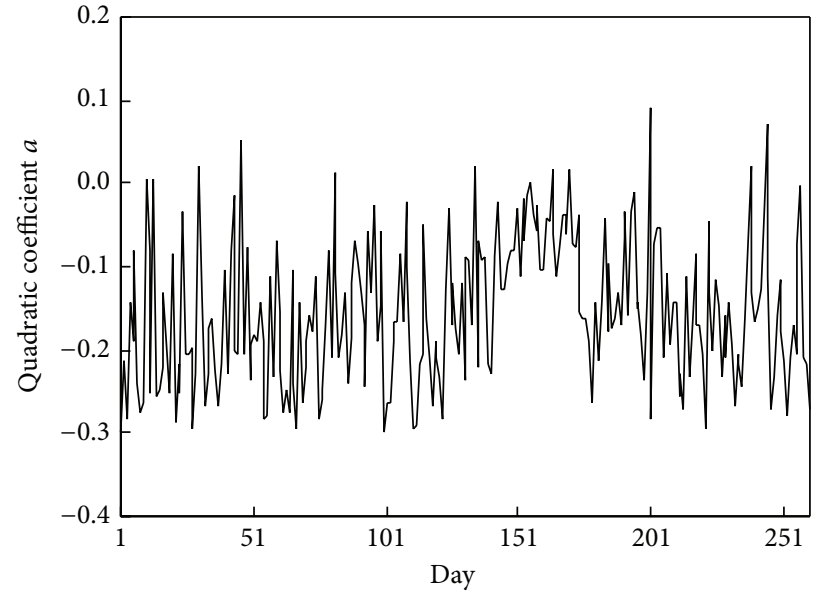

(a) Quadratic coefficient $a$

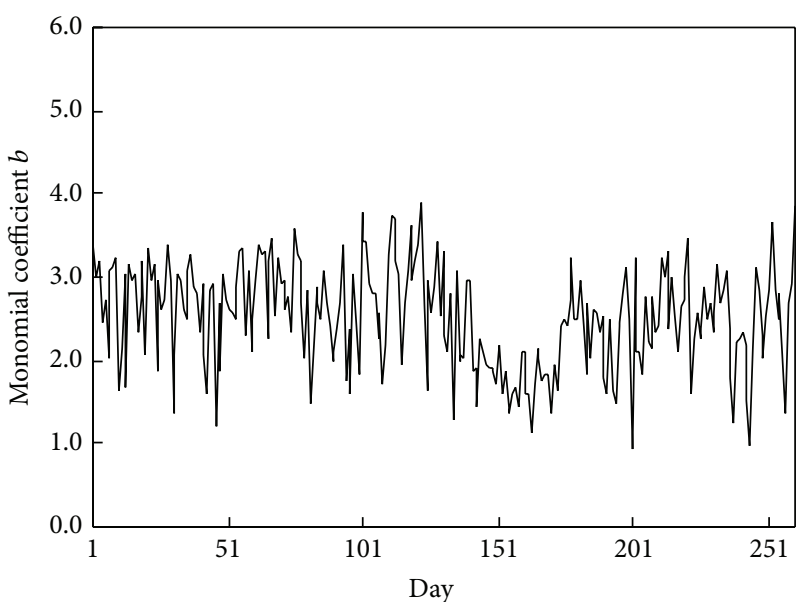

(b) Monomial coefficient $b$

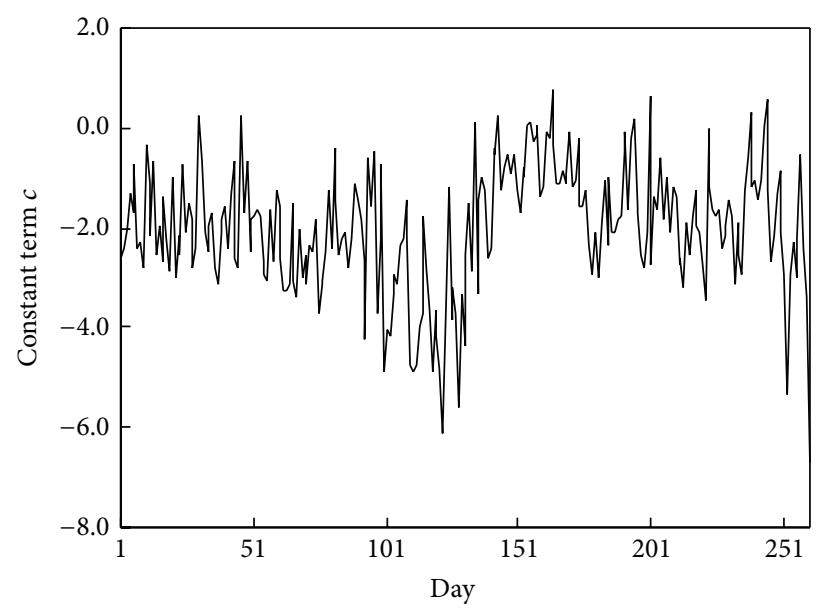

(c) Constant term $c$

FIGURE 6: Long-term monitoring results of the fitted coefficients of the quadratic polynomial.

The analysis results indicate that good cross-correlation exists between the RMS values of the transverse accelerations on the two main spans. This is because the RMS values of the transverse accelerations represent the vibration energy of the train-railway-bridge system, and the cross-correlation between the RMS values of the accelerations at different measuring positions can represent the spatial distribution of the transverse vibration energy which is inputted into the system by the traveling trains. In this case, the randomness of transverse vibration can be transferred into the deterministic feature of the spatial distribution of the transverse vibration energy. Hence, the cross-correlation model of RMS values of transverse accelerations can represent the transverse vibration state of the system when the high-speed train passes through the bridge. According to the theory of random energy analysis for train derailment [14], a stable transverse-vibration state of the train-railway-bridge system indicates a stable cross-correlation model of RMS values of the transverse accelerations. Hence, a long-term monitoring on the cross-correlation model of RMS values of the transverse accelerations can detect the abnormal changes of the cross-correlation model and can further realize the earlywarning of train running safety.

3.3. Warning Results of Train Running Safety of DaShengGuan Bridge. Based on the results in Section 3.2, the RMS value of the transverse acceleration of the main girder can be a monitoring parameter, and the cross-correlation model of the RMS values of the transverse accelerations from different measuring points can be an evaluation model for the train running safety. This section will discuss the earlywarning method of train running safety for high-speed railway bridges.

Firstly, there is a need to study the fitted coefficient of the quadratic polynomial for the cross-correlation model based on the long-term monitoring. Figure 6 provides the fitted coefficients of the quadratic polynomial for the crosscorrelation between the RMS values of the transverse accelerations in the middle of two main spans with the quadratic coefficient $a$, monomial coefficient $b$, and constant term $c$, respectively. It can be observed that although the measurements of the transverse accelerations in every single day can 
fit a quadratic polynomial model with a good correlation, the coefficients of the quadratic polynomials vary very much from a long-term point of view. This is because of the fact that, with the environmental effects such as temperature and so forth, the transverse vibration characteristics of the train-railway-bridge system will vary in a broad range. These environmental effects will cause changes in three fitted coefficients which may mask the abnormal changes caused by structural deterioration of the bridge. However, due to the diversity of the environmental effects and the complex effects on the transverse vibrations of the bridge, it is hard to accurately measure each environmental effect and to quantitatively remove the influence of each effect. For this reason, this paper investigates the normalization of environmental effects on the three fitted coefficients of the quadratic polynomial models based on PCA method. PCA method theoretically does not need to monitor the variants of environmental effects, so it has a promising prospect of engineering application [15].

Principal component analysis (PCA) method is a statistical procedure that uses an orthogonal transformation to convert a set of observations of possibly correlated variables into a set of values of linearly uncorrelated variables [15]. This method can realize the reduction treatment from a high dimension to a lower one to reveal the key information from the data by maintaining the most information. It is especially suitable for finding the hidden features in multivariant factor analysis.

The basic theory of PCA is that a vector $\mathbf{u}=$ $\left[\begin{array}{llll}u_{1} & u_{2} & \cdots & u_{n}\end{array}\right]^{\mathrm{T}}$ is operated by the dimension reduction to be a low dimension vector $\mathbf{v}=\left[\begin{array}{llll}v_{1} & v_{2} & \cdots & v_{n}\end{array}\right]^{\mathrm{T}}(m<n)$ as follows:

$$
\mathbf{v}_{m \times 1}=\mathbf{A}_{m \times n} \mathbf{u}_{n \times 1},
$$

where $\mathbf{A}$ is thetransform matrix. The $m$ components $v_{1}, v_{2}, \ldots, v_{m}$ are principal components. The principal component analysis is to find $m$ principal components which can represent more than $(80-90) \%$ of the total variance to construct a new vector $\mathbf{v}$. This vector $\mathbf{v}$ contains the most information of vector $\mathbf{u}$, so $\mathbf{v}$ realizes the condensation of the original $\mathbf{u}$. For the purpose of this paper, there is a need to transform the 3-dimensional vector $\mathbf{u}$ constructed by the three fitted coefficients of the quadratic polynomial model to a 1-dimensional vector $\mathbf{v}$. Results of the computations show that when the samples are enough, the cumulative variance of first principal component can reach $90 \%$.

According to the basic theory of PCA as stated above, the key steps of the warning method of train running safety are listed as follows.

(1) Compute the fitted coefficients of the quadratic polynomial for the RMS values of the transverse accelerations each day under the normal running condition. The three coefficients form the 3-dimensional vector $\mathbf{u}$.

(2) Reduce the dimension of the 3-dimensional vector u constructed by using $k$-day measurement to 1-dimension vector $\mathbf{v}$, where $\mathbf{A}$ is the transform matrix. The transform

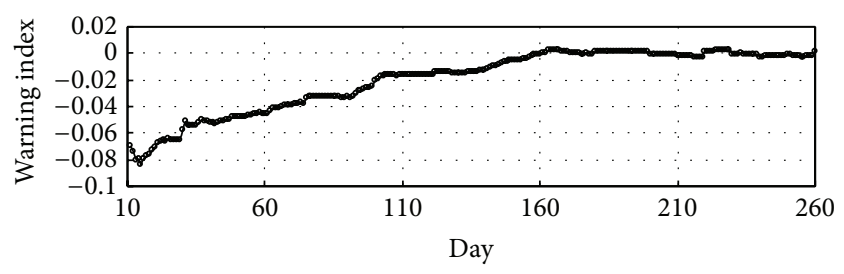

FIGURE 7: Training effect of warning idex for train running safety using PCA method.

matrix A can represent the features of the actual environmental effects so as to effectively remove the environmental effects on the fitted coefficients.

(3) The reconstructed value of the RMS of the transverse accelerations is obtained by referring to the 1-dimensional vector $\mathbf{v}$, so the warning index $\mathbf{D}$ for train running safety can be defined as follows:

$$
D(k)=v_{m}(k)-v_{e}(k),
$$

where $v_{m}(k)$ is the daily mean value of the RMS values of the transverse accelerations on the $k$ th day; $v_{e}(k)$ is the daily mean value of the RMS values of the transverse accelerations on the $k$ th day after reconstruction. When the number of monitoring days $k$ is big enough, the warning index tends to be 0 .

(4) With the help of PCA, the RMS values of the transverse accelerations in an unknown running condition can be reconstructed by using the transform matrix $\mathbf{A}$ in a normal running condition and the warning index can be computed. Comparing the warning index in a normal running condition and that in an unknown running condition, if the difference of the two is significant, it can give early warning of train running safety because of an abnormal change in the transverse vibration of the main girder.

The analysis of the monitoring data of 310 days in 2013 will be carried out as an example. The first 260-day data of the 310 days will serve as the training samples, the last 50day data will serve as the test samples. Figure 7 shows the warning index obtained by using PCA method. When the days of training data are fewer, the warning index fluctuates within -0.08 to -0.04 , while with the increase of the number of training days, the warning index gradually approaches to 0 ; when the number of training days reaches 160 , the warning index tends to be stable. The measured data of the last 50 days within the 310 days in 2013 is further processed by using the principal component reconstruction. It should be noted that the transform matrix $\mathbf{A}$ for the PCA reconstruction is obtained by using the measured data of the first 260 days to carry out principal component analysis. Figure 8 shows warning index curve from 160th to 310 th days. It can be observed that, regardless of the training samples or the test samples, the warning index varies within $-0.002-0.002$. This indicates that PCA method is capable of reconstruction and prediction by fully considering the environmental effects.

There is a need to investigate the effectiveness of the proposed method applied to the early-warning of train running safety. The RMS values of the transverse acceleration 


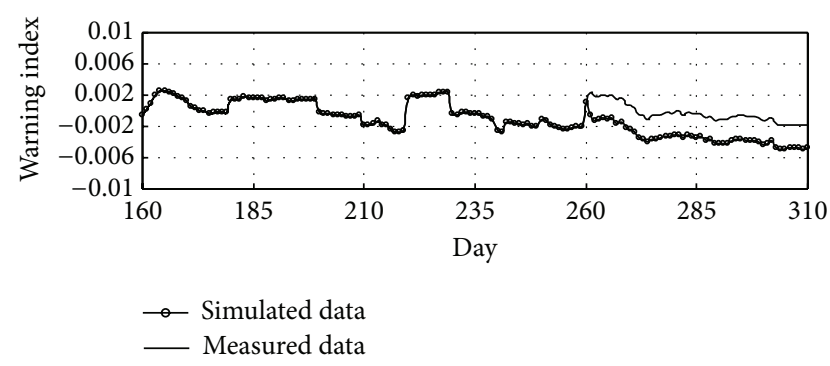

FIGURE 8: Measured waring index curve and simulated warning index curve due to the deterioration of the bridge using PCA method.

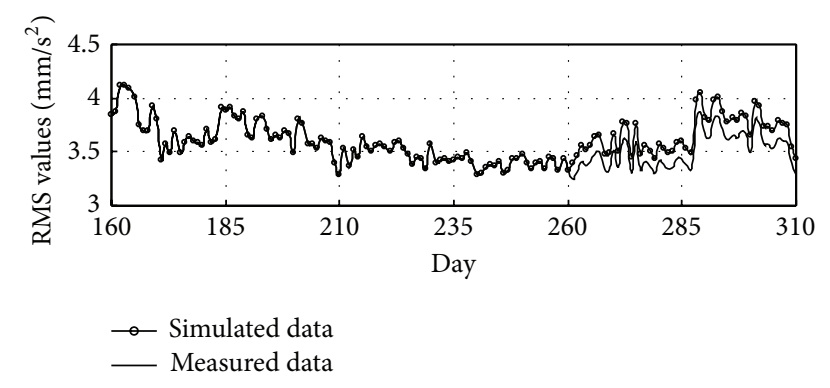

FIGURE 9: Daily mean values of the measured data and the simulated data of the RMS values of the transverse acceleration data in the middle of the second main span.

data (the data of the last 50 days in 2013) in the middle of the second main span are amplified by a factor of 1.05 to simulate the effects of structural deterioration of the bridge on the RMS values of the transverse acceleration. Figure 9 shows the daily mean values of the measured data and the simulated data of the RMS values of the transverse acceleration data in the middle of the second main span. It can be seen from Figure 9 that the RMS values of the transverse accelerations cannot detect the abnormal changes of the transverse accelerations. Figure 8 shows the simulated warning index curve using the simulated transverse acceleration data due to the deterioration of the bridge. The warning index can sensitively detect the abnormal changes of transverse accelerations so as to trigger the alarm for train running safety on time.

\section{Conclusions}

In this paper by referring to the Nanjing DaShengGuan Bridge, the first 6-track high-speed railway bridge with the longest span throughout the world, the early-warning method of train running safety of the high-speed railway bridge is proposed by adopting principal component analysis (PCA) method based on long-term transverse vibration monitoring data. The following are the findings and conclusions.

(1) When high-speed trains passed by, the transverse vibration of the train-railway-bridge system had a strong stochastic feature. The correlation between transverse accelerations from one measuring position on the main girder and the speed of trains cannot represent the transverse vibration condition of the whole system and thus cannot be adopted as the evaluating model for the train running safety.

(2) The correlation between the RMS values of the transverse accelerations from different measuring positions on the main girder represents the spatial distribution feature of transverse vibration energy of the whole system and can be effectively analyzed by a quadratic polynomial fitting model. Thus, the corresponding correlation model can be adopted as the evaluating model for the train running safety.

(3) The PCA method can effectively remove the environmental effects on the measured cross-correlation between the RMS values of transverse accelerations. The proposed warning method provides a good capability for detecting the abnormal changes of the measured transverse accelerations and hence it is suitable for early-warning of the train running safety of DaShengGuan Bridge.

\section{Conflict of Interests}

The authors declare that there is no conflict of interests regarding the publication of this paper.

\section{Acknowledgments}

The authors gratefully acknowledge the National Basic Research Program of China (973 Program) (no. 2015CB060000), the National Science and Technology Support Program of China (no. 2014BAG07B01), the Key Program of National Natural Science Foundation (no. 51438002), the Program of "Six Major Talent Summit" Foundation (no. 1105000268), and the Fundamental Research Funds for the Central Universities and the Innovation Plan Program for Ordinary University Graduates of Jiangsu Province in 2014 (no. KYLX_0156).

\section{References}

[1] N. Zhang, H. Xia, and W. W. Guo, "Vehicle-bridge interaction analysis under high-speed trains," Journal of Sound and Vibration, vol. 309, no. 3-5, pp. 407-425, 2008.

[2] C. Y. Xia, H. Xia, and G. de Roeck, "Dynamic response of a train-bridge system under collision loads and running safety evaluation of high-speed trains," Computers \& Structures, vol. 140, pp. 23-38, 2014.

[3] S. H. Ju, "Improvement of bridge structures to increase the safety of moving trains during earthquakes," Engineering Structures, vol. 56, pp. 501-508, 2013.

[4] T. L. Franklin, A. M. Joseph, and S. Emil, "Automated extraction and classification of thunderstorm and non-thunderstorm wind data for extreme-value analysis," Journal of Wind Engineering and Industrial Aerodynamics, vol. 97, no. 3-4, pp. 120-131, 2009.

[5] T. H. Yi, H. N. Li, and M. Gu, "Optimal sensor placement for structural health monitoring based on multiple optimization strategies," Structural Design of Tall and Special Buildings, vol. 20, no. 7, pp. 881-900, 2011.

[6] T.-H. Yi, H.-N. Li, and M. Gu, "Recent research and applications of GPS-based monitoring technology for high-rise structures," Structural Control and Health Monitoring, vol. 20, no. 5, pp. 649670, 2013. 
[7] E. Watanabe, H. Furuta, T. Yamaguchi, and M. Kano, "On longevity and monitoring technologies of bridges: a survey study by the Japanese Society of Steel Construction," Structure and Infrastructure Engineering, vol. 10, no. 4, pp. 471-491, 2014.

[8] Y. L. Ding and A. Q. Li, "Temperature-induced variations of measured modal frequencies of steel box girder for a long-span suspension bridge," International Journal of Steel Structures, vol. 11, no. 2, pp. 145-155, 2011.

[9] A. Deraemaeker, E. Reynders, G. de Roeck, and J. Kullaa, "Vibration-based structural health monitoring using outputonly measurements under changing environment," Mechanical Systems and Signal Processing, vol. 22, no. 1, pp. 34-56, 2008.

[10] Y. Q. Ni, J. M. Ko, X. G. Hua, and H. F. Zhou, "Variability of measured modal frequencies of a cable-stayed bridge under different wind conditions," Smart Structures and Systems, vol. 3, no. 3, pp. 341-356, 2007.

[11] Y. Q. Ni, X. G. Hua, K. Y. Wong, and J. M. Ko, "Assessment of bridge expansion joints using long-term displacement and temperature measurement," Journal of Performance of Constructed Facilities, vol. 21, no. 2, pp. 143-151, 2007.

[12] B. Chen, Z. W. Chen, Y. Z. Sun, and S. L. Zhao, "Condition assessment on thermal effects of a suspension bridge based on SHM oriented model and data," Mathematical Problems in Engineering, vol. 2013, Article ID 256816, 18 pages, 2013.

[13] A. D. Orcesi and D. M. Frangopol, "Bridge performance monitoring based on traffic data," Journal of Engineering Mechanics, vol. 139, no. 11, pp. 1508-1520, 2013.

[14] Q. Zeng Y, J. Xiang, P. Lou, and Z. Zhou, "Mechanical mechanism of derailment and theory of derailment prevention," Journal of Railway Science and Engineering, vol. 1, no. 1, pp. 1931, 2004.

[15] X. G. Hua, Y. Q. Ni, J. M. Ko, and K. Y. Wong, "Modeling of temperature-frequency correlation using combined principal component analysis and support vector regression technique," Journal of Computing in Civil Engineering, vol. 21, no. 2, pp. 122135, 2007. 

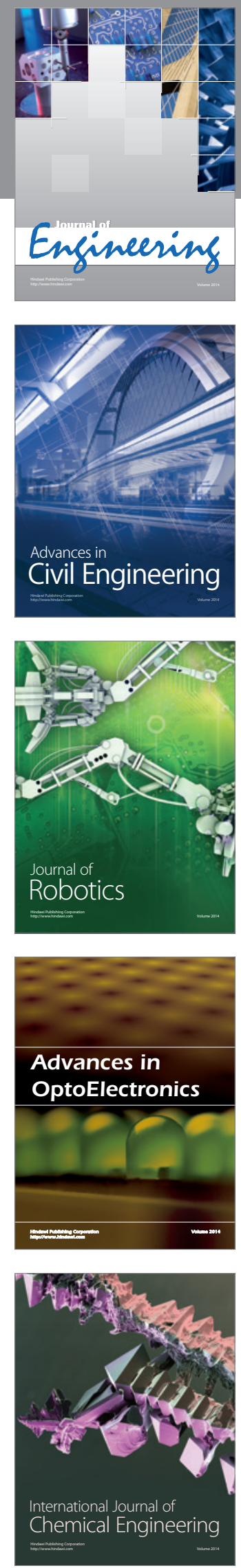

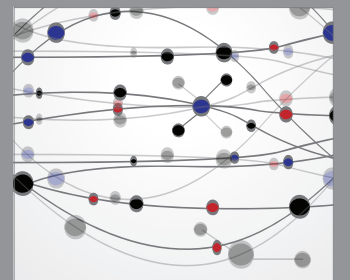

The Scientific World Journal
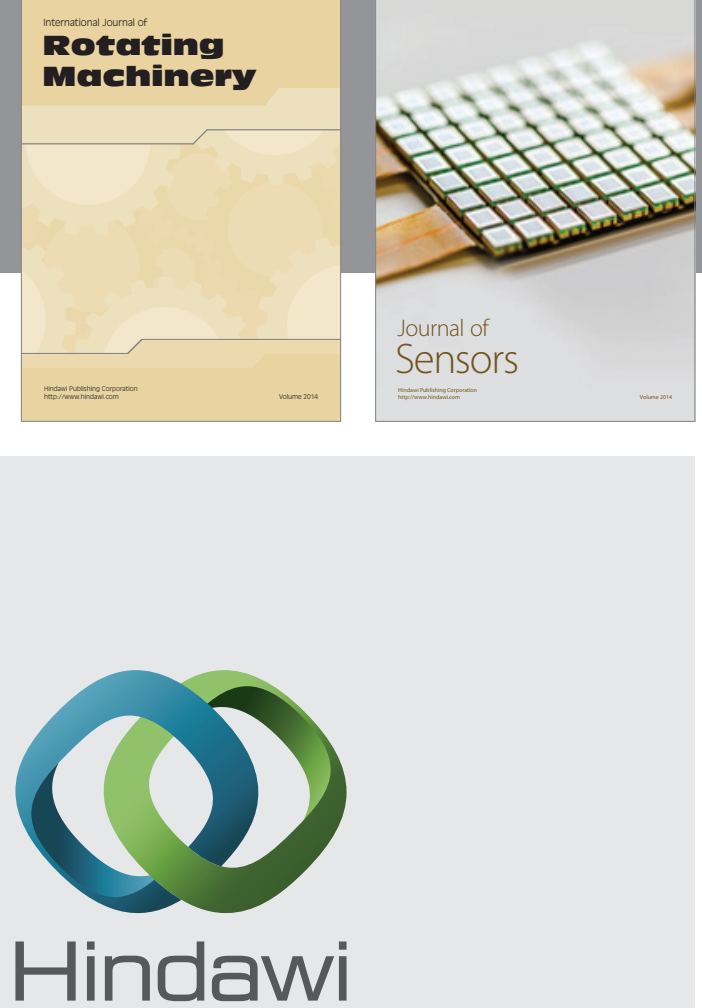

Submit your manuscripts at http://www.hindawi.com
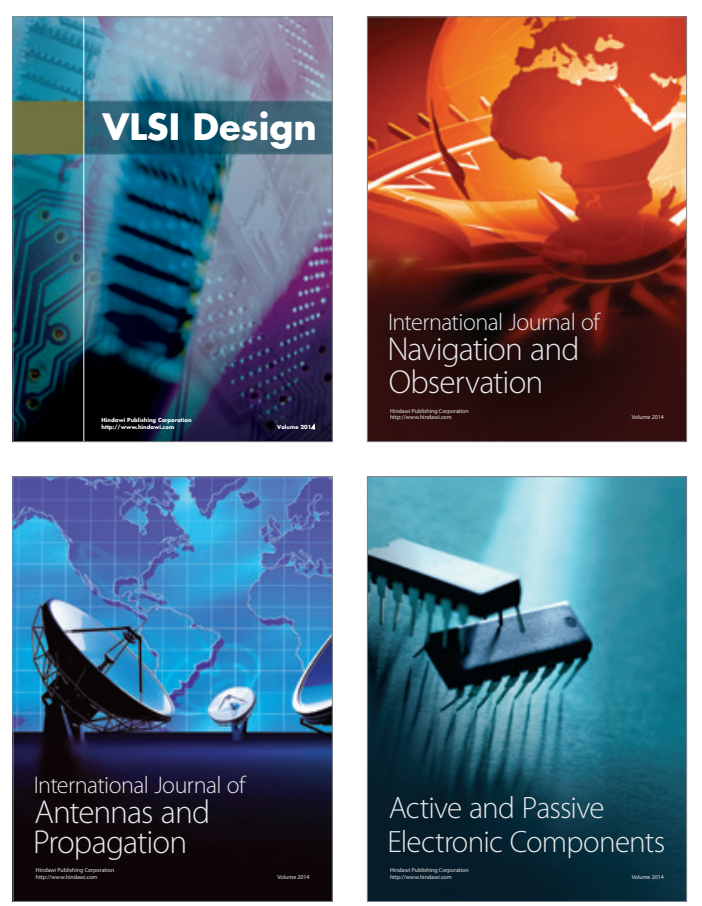
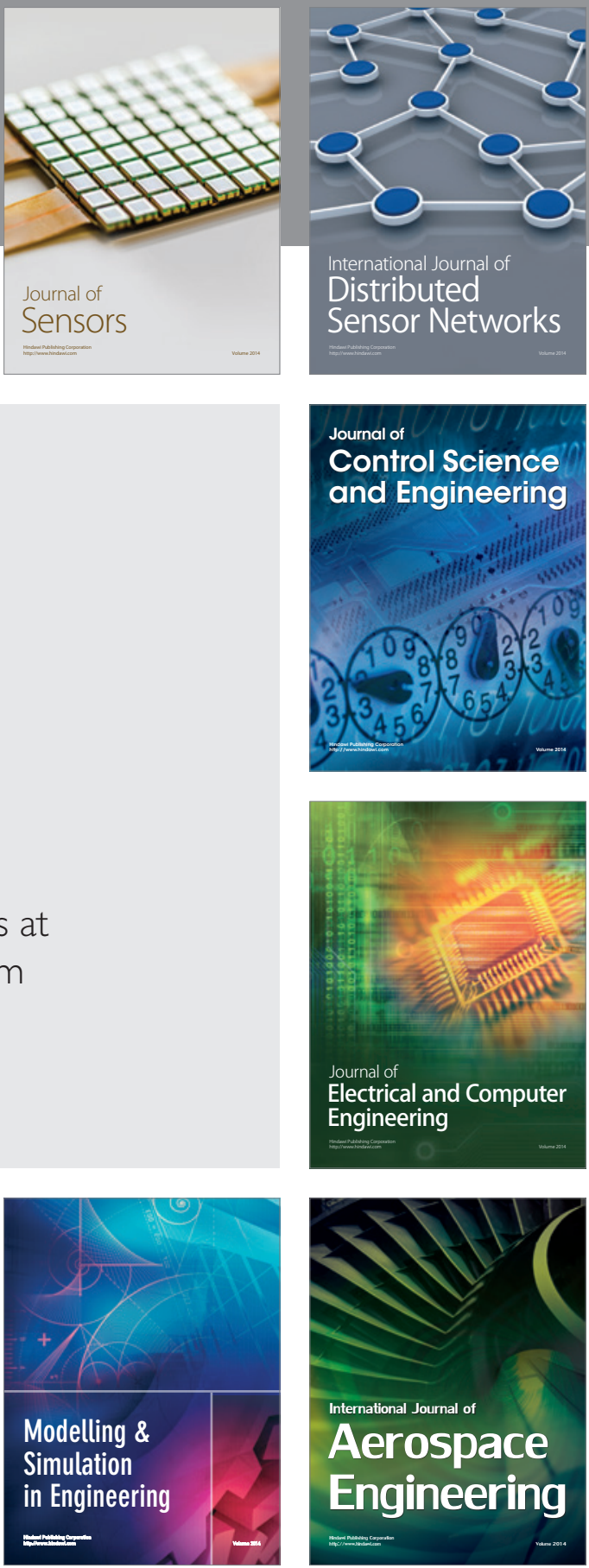

Journal of

Control Science

and Engineering
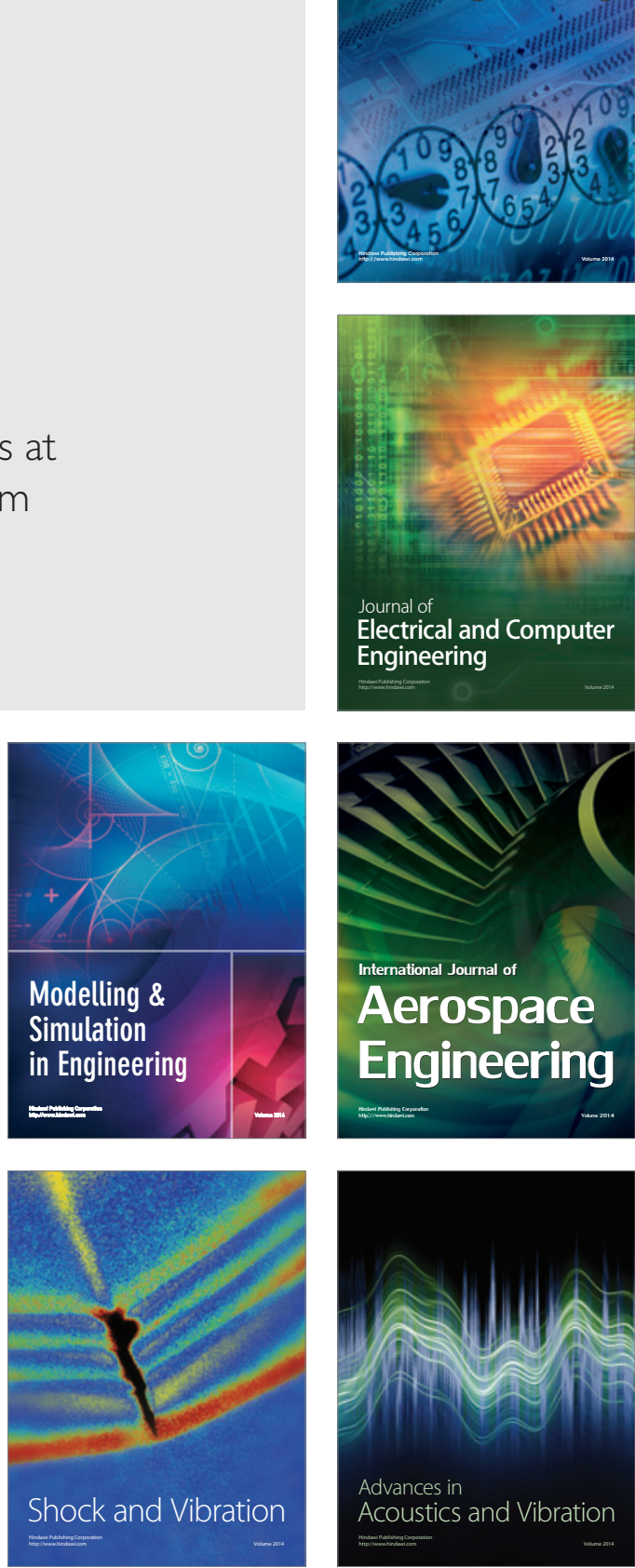